\title{
UNIVERSAL PROPERTIES OF PRYM VARIETIES WITH AN APPLICATION TO ALGEBRAIC CURVES OF GENUS FIVE
}

\author{
BY \\ LEON MASIEWICKI
}

\begin{abstract}
It is proved that every morphism of a curve with an involution into an Abelian variety, anticommuting with the involution, factors through the associated Prym variety. This result is used to show that Jacobians of curves of genus five arise as Prym varieties associated to a certain class of curves.
\end{abstract}

1. Principally polarized Abelian varieties. Let $X$ be a complex torus. We have $X=E / V$, where $E \approx C^{g}$ is the universal covering space of $X$ and $V$ is a lattice in $E$. Then $\pi_{1}(X)=H_{1}(X, Z)=V$ and $H^{*}(X, Z)=\operatorname{Hom}(\Lambda V, Z)$; where $\Lambda V$ is the exterior algebra generated by $V$ over $Z$.

Let $\Sigma$ be a theta-divisor on $X . \Sigma$ is called principal if the complete linear series of $\Sigma$ is one-dimensional, i.e., if $|\Sigma|=\operatorname{dim} H^{0}\left(O_{X}(\Sigma)\right)=1 . X$ together with a principal theta-divisor $\Sigma$ is called a principally polarized Abelian variety.

$\hat{X}=\operatorname{Pic}^{0}(X)$ is called the dual variety. A polarization $\Sigma$ of $X$ induces a homomorphism $\lambda_{\Sigma}: X \rightarrow \hat{X}$ defined by $\lambda_{\Sigma}(x)=T_{x}(\Sigma)-\Sigma$, where $T_{x}$ is the translation by $x$. It is known that if $\Sigma$ is principal, then $\lambda_{\Sigma}$ is an isomorphism and $\hat{\Sigma}-\lambda_{\Sigma}(\Sigma)$ is a principal theta-divisor on $\hat{X}[\mathbf{M 1}]$.

There exists a positive definite Hermitian form $H=\Phi+i \psi$ on $E$ such that the restriction of $\psi$ to $V \wedge V$ is integer-valued and a map $\alpha: V \rightarrow S^{1}$, the unit circle, such that $\alpha\left(u_{1}+u_{2}\right)=\alpha\left(u_{1}\right) \alpha\left(u_{2}\right) \exp \pi i \psi\left(u_{1}, u_{2}\right)$ with the property that $\Sigma$ is the divisor of zeros at the theta function $\theta(z)$ with the periodicity relations

$$
\theta(z+u)=\alpha(u) \exp \pi(H(z, u)+1 / 2 H(u, u)) \theta(z)
$$

for all $z \in E, u \in V$ (ibid.).

Note that $\psi \in \operatorname{Hom}\left(\Lambda^{2} V, Z\right)=H^{2}(X, Z)$. It is known from topology that $\psi$ is the Poincare dual of $\Sigma$, i.e., $\psi \cap(X)=[\Sigma] \in H_{2 g-2}(X, Z)$, where $(X)$ is the orientation class of $X$ and $[\Sigma]$ is the homology class represented by

Received by the editors May 5, 1975.

AMS (MOS) subject classifications (1970). Primary 14H30, 14K99, 30A52;

Secondary $14 \mathrm{H} 40,30 \mathrm{~A} 48$.

Key words and phrases. Principally polarized Abelian variety, theta divisor, Prym variety, ramified covering. 
$\Sigma$. Also, $|\Sigma|=(\operatorname{det} \psi)^{1 / 2}$. Thus $H$ induces a principal polarization of $X$ if $\psi=$ Im $H$ is unimodular (ibid.).

From now on let $(X, \Sigma)$ be a principally polarized Abelian variety.

Let $\times: H_{i}(X, Z) \otimes H_{j}(X, Z) \rightarrow H_{i+j}(X, Z)$ be the Pontriagin product, i.e., the homology operation induced by the group law on $X$ and let $\cdot: H_{i}(X, Z) \otimes$ $H_{j}(X, Z) \rightarrow H_{i+j-2 g}(X, Z)$ be the intersection product on $X . B=\left\{\sigma_{i}, \tau_{i}\right\}, i \leqslant$ $g$, is called a symplectic basis of $X$ if $B$ generates $V$ and $\psi\left(\sigma_{i}, \tau_{j}\right)=\delta_{i j}$, $\psi\left(\sigma_{i}, \sigma_{j}\right)=\psi\left(\tau_{i}, \tau_{j}\right)=0$ (or equivalently, $\left(\sigma_{i} \times \tau_{j}\right) \cdot \Sigma=\delta_{i j},\left(\sigma_{i} \times \sigma_{j}\right) \cdot \Sigma=$ $\left.\left(\tau_{i} \times \tau_{j}\right) \cdot \Sigma=0\right)$.

Let $\Sigma^{(n)}=\Sigma \cdot \cdots \cdot \Sigma(n$ times $) . \quad \Sigma^{(n)} \in H_{2 g-2 n}(X, Z)$ and

$$
\begin{gathered}
\Sigma^{(0)}=(X), \quad \Sigma^{(1)}=[\Sigma]=\sum_{i=1}^{g} \prod_{j \neq i}\left(\sigma_{j} \times \tau_{j}\right), \\
\Sigma^{(g-1)}=(g-1) ! \sum_{i=1}^{g} \sigma_{i} \times \tau_{i} \quad \text { and } \quad \Sigma^{(g)}=g ! \in H_{0}(X, Z) .
\end{gathered}
$$

One has the Hodge decomposition of $H^{1}(X, Z) \otimes C$

$$
H(X, Z) \otimes C=H^{1,0}(X) \oplus H^{0,1}(X)
$$

inducing the Hodge decomposition

$$
H^{2}(X, Z) \otimes C=H^{2,0}(X)+H^{1,1}(X)+H^{0,2}(X),
$$

where $H^{i j}(X)=\Lambda^{i} H^{1,0}(X) \otimes_{C} \Lambda^{j} H^{0,1}(X)$.

Let $\left\{\omega_{i}, \eta_{i}\right\}, i=1, \ldots, g$, be the basis of $H^{1}(X, Z)$ dual to a symplectic basis $B$ of $X$. We can choose $\omega_{i} \in H^{1,0}(X)$. Then $H^{i, j}(X)$ has a basis $\left\{\omega_{k_{1}} \wedge\right.$ $\left.\cdots \wedge \omega_{k_{i}} \otimes \bar{\omega}_{l_{1}} \wedge \bar{\omega}_{l_{2}} \wedge \ldots \wedge \omega_{l_{j}}\right\}, k_{1}<k_{2}<\cdots<k_{i}, l_{1}<l_{2}<\cdots<l_{j}$.

(1.2) It follows that $E=H^{1,0}(X)$ and

$$
H(\alpha, \beta)=\frac{1}{(g-1) !} \int_{\Sigma}(g-1) \alpha \wedge \bar{\beta}
$$

Also, $\psi=\sum_{i=1}^{g} \omega_{i} \otimes \bar{\omega}_{i} \in H^{1,1}(X) \cap H^{2}(X, Z)$ (compare [M1] , [CG]). If $E=E^{(1)} \oplus E^{(2)}$ is a direct sum decomposition such that $V^{(i)}=E^{(i)}$ $\cap V$ is a lattice in $E^{(i)}$ for $i=1,2 ; V=V^{(1)} \oplus V^{(2)}$ and $E^{(1)} \perp E^{(2)}$ (i.e., $\left.H(\alpha, \beta)=0 \forall \alpha \in E^{(1)}, \beta \in E^{(2)}\right)$, then $X$ has a decomposition $X=X^{(1)} \times$ $X^{(2)}$ such that $H^{(i)}=H \mid E^{(i)}$ is a positive definite Hermitian form on $E^{(i)}$ inducing a principal polarization $\Sigma^{(i)}$ on $X^{(i)}=E^{(i)} / V^{(i)}$. Then $\Sigma=X^{(1)} \times$ $\Sigma^{(2)}+\Sigma^{(1)} \times X^{(2)}$ is reducible unless $E^{(1)}$ or $E^{(2)}$ is trivial [CG].

(1.3) $V^{1}$ is a direct summand of $V$ if and only if the inclusion homomorphism $V^{1} \hookrightarrow V$ has a left inverse. Then, choosing bases $B^{1}$ and $B$ in $V$ and $V^{1}$, 
we have the matrix $M_{B}^{B}{ }^{1}$ (incl) which is left-invertible over $Z$. This happens if and only if $M_{B}^{B^{1}}$ (incl) has an $n \times n$ unimodular minor, where $n=\mathrm{rk} V^{1}$.

2. Jacobi varieties and Prym varieties. Let $\pi: \widetilde{C} \rightarrow C$ be an unramified two-sheeted covering of a smooth complete algebraic curve $C$ of positive genus $g$. Let $\iota: \widetilde{C} \rightarrow \widetilde{C}$ be the deck transformation of $\pi$ and let $\pi^{*}: J(C) \rightarrow J(\widetilde{C}), \mathrm{Nm}$ : $J(\tilde{C}) \rightarrow J(C)$ and $\iota: J(\widetilde{C}) \rightarrow J(\widetilde{C})$ be the induced homomorphisms of Jacobi varieties.

$J(C)=H^{1,0}(C)^{*} / H_{1}(C, Z)$ is a $g$-dimensional complex torus with principal polarization $\Theta$ given by the Riemann's theta function $\theta(Z)$. The Poincare dual of $\Theta$ is the intersection pairing on $C\langle\rangle:, H_{1}(C, Z) \wedge H_{1}(C, Z) \rightarrow Z . H_{1}(C, Z)$ has a canonical basis $B=\left\{\gamma_{i}, \delta_{i}\right\}, i \leqslant g$, such that $\left\langle\gamma_{i}, \delta_{j}\right\rangle=\delta_{i j},\left\langle\gamma_{i}, \gamma_{j}\right\rangle=$ $\left\langle\delta_{i}, \delta_{j}\right\rangle=0$. Thus $B$ is a symplectic basis of $J(C)$. Note that the intersection pairing is represented in the basis $B$ by the matrix $\left[\begin{array}{cc}0 & I_{0}^{I d} g\end{array}\right]$, and is therefore unimodular. If we extend the form $\langle$,$\rangle to H^{1,0}(C)^{*}$ by $R$-linearity, there exists a unique positive definite Hermitian form $H$ such that $\operatorname{Im} H=\langle\rangle .$,$H is given$ by the formula $H(\alpha, \beta)=\langle i \alpha, \beta\rangle+i\langle\alpha, \beta\rangle$.

The homology class $(1 /(g-n) !) \Theta^{(g-n)}$ is represented by the image of the $n$th symmetric product of $C$ under the standard map $t: C \rightarrow J(C)$ sending $P \in$ $C$ to $\int_{P_{0}}^{P}-\bmod H_{1}(C, Z)$, where $P_{0}$ is the base point.

Similarly, $H_{1}(\widetilde{C}, Z)$ has a canonical basis $\widetilde{B}=\left\{\gamma_{i}^{(\alpha)}, \delta_{i}^{(\alpha)}, \lambda, \mu\right\}, i<g$; $\alpha=0,1$. $\iota$ acts on $H_{1}(\widetilde{C}, Z)$ by $\iota\left(\gamma_{i}^{(\alpha)}\right)=\gamma_{i}^{(1-\alpha)}, \iota\left(\delta_{i}^{(\alpha)}\right)=\delta_{i}^{(1-\alpha)}, \iota(\lambda)=\lambda$ and $\iota(\mu)=\mu$. Also, $\pi: H_{1}(\widetilde{C}, Z) \rightarrow H_{1}(C, Z)$ sends $\gamma_{i}^{(\alpha)}$ to $\gamma_{i}, \delta_{i}^{(\alpha)}$ to $\delta_{i}$ for $i<6, \pi(\lambda)=\gamma_{g}, \pi(\mu)=2 \delta_{g}$.

The following properties of $\mathrm{Nm}, \pi^{*}$ and $\iota$ follow directly from the definition:

(2.1) $\mathrm{Nm} \circ \pi^{*}=2 \mathrm{Id} J(C)$ and therefore $\mathrm{Nm}$ is surjective.

(2.2) $\pi^{*} \circ \mathrm{Nm}=\mathrm{Id}_{J(\widetilde{C})}+\iota$.

(2.3) $\iota \circ \pi^{*}=\pi^{*}$.

(2.4) $\pi^{*} J(C)=\{x \in J(\tilde{C}): x=\varkappa\}$.

It is convenient to consider the involution induced by $\iota$ on $H^{1,0}(\tilde{C})^{*}$. By (2.4), เ has a $g$-dimensional 1-eigenspace in $H^{1,0}(\widetilde{C})^{*}$. Since $\operatorname{dim} H^{1,0}(\widetilde{C})^{*}=$ $2 g-1$ by Riemann-Hurwitz relation and $\iota^{2}=I d, \iota$ has a -1 eigenspace $E$ with $\operatorname{dim} E=g-1$.

Let $V \subset H_{1}(\widetilde{C}, Z)$ be the subgroup generated by $C=\left\{\gamma_{i}^{(0)}-\gamma_{i}^{(1)}, \delta_{i}^{(0)}-\right.$ $\left.\delta_{i}^{(1)}\right\}, i<g . \quad V$ is a free Abelian group of rank $2 g-2$ contained in $E$. As a matter of fact, $V=E \cap H_{1}(\widetilde{C}, Z)$ is the largest subgroup of $H_{1}(\widetilde{C}, Z)$ on which $\iota$ acts as minus identity. Moreover, since the elements of $C$ are linearly independent over $R, V$ is a lattice in $E$. Thus $E / V$ is a $g-1$-dimensional complex torus.

The intersection pairing on $\widetilde{C}$ pulls back to a bilinear form $\chi$ on $V$ such that 


$$
\begin{aligned}
& \chi\left(\gamma_{i}^{(0)}-\gamma_{i}^{(1)}, \delta_{j}^{(0)}-\delta_{j}^{(1)}\right)=2 \delta_{i j} \\
& \chi\left(\gamma_{i}^{(0)}-\gamma_{i}^{(1)}, \gamma_{j}^{(0)}-\gamma_{j}^{(1)}\right)=\chi\left(\delta_{i}^{(0)}-\delta_{i}^{(1)}, \delta_{j}^{(0)}-\delta_{j}^{(1)}\right)=0
\end{aligned}
$$

$x \in \operatorname{Hom}\left(\Lambda^{2} V, Z\right)=H^{2}(E / V, Z)$ is represented in the basis $C$ by the matrix

$$
\left[\begin{array}{cc}
0 & 2 \mathrm{Id}_{g-1} \\
-2 \mathrm{Id}_{g-1} & 0
\end{array}\right],
$$

so $1 / 2 \chi \in H^{2}(E / V, Z)$ is unimodular, and so it induces a principal polarization $\Xi$ of $E / V$ such that $C$ is a symplectic basis.

In the language of theta-divisors, the principal polarization $\Xi$ is given by the divisor of the theta function $(\tilde{\theta} \mid E)^{1 / 2}$ on $E / V$.

(2.5) Definition. $P=E / V$ with the principal polarization described above is called the Prym variety associated to the unramified two-sheeted covering $\pi: \widetilde{C} \rightarrow C$.

It is clear from the construction that

$$
P=\operatorname{ker} \mathrm{Nm}^{0}=\operatorname{ker}\left(\operatorname{Id}_{J(\tilde{C})}+\imath\right)^{0}=\operatorname{Im}\left(\operatorname{Id}_{J(\tilde{C})}-\imath\right)
$$

where $A^{0}$ denotes the connected component of $A \subset J(\tilde{C})$ containing the origin.

Let $\alpha: J(\tilde{C}) \rightarrow P$ be the epimorphism given by $\alpha(x)=x-\omega$ and let $\tilde{t}$ : $\widetilde{C} \rightarrow J(\widetilde{C})$ be the standard imbedding with the basepoint $P_{0}$.

(2.7) Proposition. $\alpha \circ \tilde{t}: \widetilde{C} \rightarrow P$ is one to one unless both $\widetilde{C}$ and $C$ are hyperelliptic.

Proof. $\tilde{t}(P) \in J(\tilde{C})=\operatorname{Pic}^{0}(\tilde{C})$ represents the divisor $P-P_{0}$. Thus $\alpha \circ$ $\tilde{t}(P)=\alpha \circ \tilde{t}(Q)$ implies by Abel's Theorem that $P-P=Q-Q$ and therefore $P+\iota=Q+\iota P$. Since $\iota$ has no fixed points, we have $P=Q$ or $\widetilde{C}$ is hyperelliptic. It now suffices to prove that if $\widetilde{C}$ is hyperelliptic, then so is $C$. Let $j$ be the hyperelliptic involution on $\widetilde{C}$. Since $j$ commutes with every automorphism of $\widetilde{C}$, we have $j \iota=\imath$, so that $j$ induces an involution $j_{c}$ on $C$.

$j$ has $4 g=(2(2 g-1)+2)$ fixed points. Note that if $P \in \widetilde{C}$ is a fixed point of $j$, then so is $i P$ and $\pi(P)$ is a fixed point of $j_{C}$. Thus $j_{C}$ has at least $2 g$ fixed points. Let $k$ be the genus of the quotient curve $C / j_{C}$. Then by RiemannHurwitz Relation, $2(2 k-2)+2 g \leqslant 2 g-2$, so that $4 k \leqslant 2$ which implies $k=0$. Thus $C$ is a two-sheeted covering of a rational curve and therefore is hyperelliptic.

3. Universal properties. Throughout this section let $P$ be the Prym variety associated to an unramified two-sheeted covering $\pi: \widetilde{C} \rightarrow C$ of a smooth complete algebraic curve $C$ of genus $g>0$.

(3.1) Proposition. Let $X$ be an Abelian variety with a principal theta- 
divisor $\Sigma$. If $\varphi: \widetilde{C} \rightarrow X$ is a map such that $\varphi \circ \iota=-\varphi$, then there exists a unique homomorphism $\psi: P \rightarrow X$ such that $\varphi=\psi \circ \alpha \circ \tilde{t}$.

Proof. Since $J(\widetilde{C})$ is the Albanese variety of $\widetilde{C}$, there exists a homomorphism $\bar{\varphi}: J(\widetilde{C}) \rightarrow X$ such that $\bar{\varphi} \circ \tilde{t}=\varphi$ and $\bar{\varphi} \circ \iota=-\bar{\varphi}$. Therefore $\bar{\varphi} \circ \alpha=$ $\bar{\varphi}(\operatorname{Id} J(\tilde{C})-\iota)=2 \bar{\varphi}$, so that we have a commutative diagram:

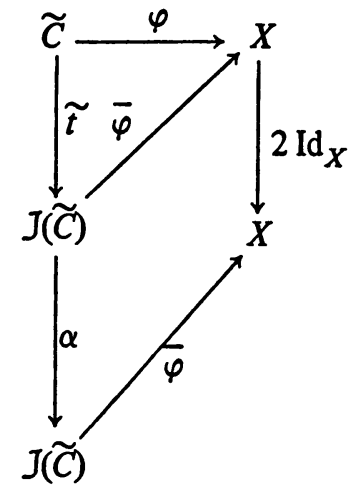

Put $\bar{\psi}=\bar{\varphi}|\operatorname{Im} \alpha=\bar{\varphi}| P: P \rightarrow X$. Then $\bar{\psi} \circ \alpha=2 \bar{\varphi}: J(\tilde{C}) \rightarrow X$, so that the following diagram commutes:

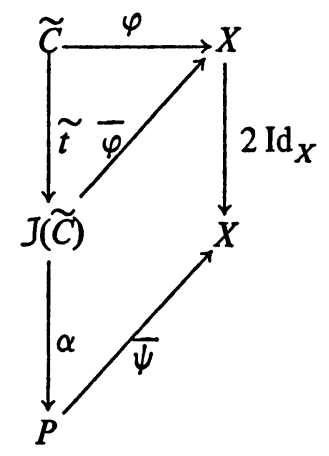

Let $x$ be a point of order 2 in $P$. If $y \in J(\widetilde{C})$ is such that $\alpha(y)=x$, then $2 \alpha(y)$ $=0$ so that $u(2 y)=2 y$. Thus there is $u \in J(C)$ such that $\pi^{*} u=2 y$ and $v \in$ $J(C)$ such that $2 v=u$. Since $2\left(\pi^{*} v-y\right)=0, \pi^{*} v-y$ is an element of order two in $J(\widetilde{C})$. We have $\alpha\left(\pi^{*} v-y\right)=\pi^{*} v-y-\imath \pi^{*} v+\iota y=-(y-\imath y)=$ $-\alpha(y)=-x=x$ because $\pi^{*} v=\imath \pi^{*} v$ by (2.3). Let $X_{2}$ denote the subgroup of elements of order 2 of the Abelian variety $X$. We have proved that $\alpha_{2}: J(\widetilde{C})_{2}$ $\rightarrow P_{2}$ is an epimorphism.

Let $x \in P_{2}$ and $y \in J(\widetilde{C})_{2}$ with $\alpha(y)=x$. Then $\bar{\psi}(x)=\bar{\varphi} \circ \alpha(y)=$ $2 \bar{\varphi}(y)=\bar{\varphi}(2 y)=0$, so that $\bar{\psi}$ factors through 2 Id $_{P}$ by elementary algebra. Thus there exists a homomorphism $\psi: P \rightarrow X$ such that $\bar{\psi}=\psi \circ 2 \operatorname{Id}_{P}=$ $\operatorname{2Id}_{X}^{\circ} \psi$.

We know that $2 \psi \circ \alpha=2 \bar{\varphi}: J(\tilde{C}) \rightarrow X$. Therefore $\psi \circ \alpha \circ 2 \operatorname{Id}_{\mathrm{J}(\tilde{C})}=$ 
$\bar{\varphi} \circ 2 \operatorname{Id} J(\tilde{C})$ and so $\psi \circ \alpha=\bar{\varphi}$ because $2 \operatorname{Id} J(\tilde{C})$ is an epimorphism. Finally, $\psi \circ \alpha \circ \widetilde{t}=\bar{\varphi} \circ \tilde{t}=\varphi$, as claimed.

If $\psi^{\prime}$ is another such homomorphism, we have $\psi^{\prime} \circ \alpha=\bar{\varphi}=\psi \circ \alpha$ : J( $\left.\tilde{C}\right)$ $\rightarrow X$. But $\alpha: J(\widetilde{C}) \rightarrow P$ is onto, so that $\psi^{\prime}=\psi$, proving uniqueness.

(3.2) LEMMA. $\alpha \circ \tilde{t}(\widetilde{C})$ represents the homology class $(2 /(g-2) !) \Xi(g-2)$ in $\mathrm{H}_{2}(P, Z)$.

Proof. Let the symplectic bases $\widetilde{B}$ and $C$ be as before. Then $[t(\widetilde{C})]=$ $\lambda \times \mu+\Sigma_{i, \alpha} \gamma_{i}^{(\alpha)} \times \delta_{i}^{(\alpha)}$ by $(1.1)$ and because $[t(\widetilde{C})]=(1 /(2 g-2) !) \tilde{\partial}^{(2 g-2)}$. Hence

$$
\begin{aligned}
{[\alpha \circ t(\widetilde{C})] } & =\alpha(\lambda \times \mu)+\sum_{i=1}^{g-1} \alpha\left(\gamma_{i}^{(0)} \times \delta_{i}^{(0)}\right)+\alpha\left(\gamma_{i}^{(1)} \times \delta_{i}^{(1)}\right) \\
& =\sum_{i=1}^{g-1} \alpha\left(\gamma_{i}^{(0)}\right) \times \alpha\left(\delta_{i}^{(0)}\right)+\alpha\left(\gamma_{i}^{(1)}\right) \times \alpha\left(\delta_{i}^{(1)}\right) \\
& =\sum_{i=1}^{g-1}\left(\gamma_{i}^{(0)}-\gamma_{i}^{(1)}\right) \times\left(\delta_{i}^{(0)}-\delta_{i}^{(1)}\right)+\left(\gamma_{i}^{(1)}-\gamma_{i}^{(0)}\right) \times\left(\delta_{i}^{(1)}-\delta_{i}^{(0)}\right) \\
& =2 \sum_{i=1}^{g-1}\left(\gamma_{i}^{(0)}-\gamma_{i}^{(1)}\right) \times\left(\delta_{i}^{(0)}-\delta_{i}^{(1)}\right) \\
& =2 \cdot \frac{1}{(g-2) !} \Xi^{(g-2)}
\end{aligned}
$$

because $C$ is a symplectic basis of $P$ and $\alpha \lambda=\alpha \mu=0$.

(3.3) THEOREM. With the hypotheses of Proposition (3.1), if $\varphi(\widetilde{C})$ represents the homology class $(2 / n !) \Sigma^{(n)}$ (where $\left.\operatorname{dim} X=n+1\right)$, then $X$ is isomorphic to a direct factor of $P$, so that either $X \approx P$ (as principally polarized Abelian varieties) or $P$ is reducible.

PRoof. $\psi: P \rightarrow X$ maps $\alpha \circ \tilde{t}(\tilde{C})$ to a cycle representing $(2 / n !) \Sigma^{(n)}$. Thus the induced homology homomorphism $\psi_{*}: H_{2}(P, Z) \rightarrow H_{2}(X, Z)$ sends $(2 /(g-2) !) \Xi^{(g-2)}$ to $(2 / n !) \Sigma^{(n)}$ by Lemma (3.2). We have therefore

$$
2\left(\psi_{*}\left(\frac{1}{(g-2) !} \Xi^{(g-2)}\right)-\frac{1}{n !} \Sigma^{(n)}\right)=0
$$

and since homology of a torus is torsion free,

$$
\psi_{*}\left(\frac{1}{(g-2) !} \Xi^{(g-2)}\right)=\frac{1}{n !} \Sigma^{(n)} .
$$

Let us compare Hermitian forms 


$$
H_{\Xi}(\omega, \eta)=\int_{(1 /(g-2) !) \Xi^{(g-2)}} \omega \wedge \bar{\eta} \quad \text { on } H^{1,0}(P)
$$

and

We have

$$
H_{\Sigma}(\alpha, \beta)=\int_{(1 / n !) \Sigma^{(n)}}^{\alpha \wedge \bar{\beta}} \text { on } H^{1,0}(X)
$$

$$
\begin{aligned}
H_{\Xi}\left(\psi^{*} \alpha, \psi^{*} \beta\right) & =\int_{(1 /(g-2) !) \Xi}(g-2) \psi^{*} \alpha \wedge \overline{\psi^{*} \beta} \\
& =\int_{\psi_{*}\left((1 / n !) \Sigma^{(n)}\right)} \psi^{*}(\alpha \wedge \bar{\beta}) \\
& =\int_{(1 / n !) \Sigma^{(n)}} \alpha \wedge \bar{\beta}=H_{\Sigma}(\alpha, \beta)
\end{aligned}
$$

If $\psi: P \rightarrow X$ is not onto, there exists a nonzero $\alpha \in H^{1,0}(X)$ such that $\psi^{*} \alpha=0 \in H^{1,0}(P)$. Then for every $\beta \in H^{1,0}(X)$ we have

$$
H_{\Sigma}(\alpha, \beta)=H_{\Xi}\left(\psi^{*} \alpha, \psi^{*} \beta\right)=H_{\Xi}(0, \beta)=0
$$

by (3.4) which contradicts nondegeneracy of $H_{\Sigma}$.

Consider now dual varieties $\hat{P}=\operatorname{Pic}^{0}(P)=H^{1,0}(P) / H^{1}(P, Z)$ and $\hat{X}=$ $H^{1,0}(X) / H^{1}(X, Z)$. Recall that a principally polarized Abelian variety is isomorphic to its dual.

There is the induced monomorphism $\hat{\psi}=\psi^{*}: \hat{X} \rightarrow \hat{P}$. It suffices to prove that $\hat{P}=\hat{\psi}(\hat{X}) \times \hat{Y}$, where $Y$ is an Abelian variety. We begin by proving that $\psi^{*}\left(H^{1}(X, Z)\right)$ is a direct summand of $H^{1}(P, Z)$. Recall that $\Psi_{\Sigma}=\operatorname{Im} H_{\Sigma}$ has integer values on $H^{1}(X, Z)$, and similarly $\Psi_{\Xi}=\operatorname{Im} H_{\Xi} \in H^{2}(P, Z)$.

Let $\left\{\alpha_{i}, \beta_{i}\right\}, i \leqslant n+1$, be a basis of $H^{1}(X, Z)$ such that

$$
\begin{aligned}
& \Psi_{\Sigma}\left(\alpha_{i}, \beta_{j}\right)=\operatorname{Im} \int_{(1 / n !) \Sigma}(n) \alpha_{i} \wedge \bar{\beta}_{j}=\delta_{i j} \\
& \Psi_{\Sigma}\left(\alpha_{i}, \alpha_{j}\right)=\Psi_{\Sigma}\left(\beta_{i}, \beta_{j}\right)=0 .
\end{aligned}
$$

$\left\{\alpha_{i}, \beta_{i}\right\}$ is dual of a symplectic basis of $X$ and is a symplectic basis of $\hat{X}$. $\psi^{*} H^{1}(X, Z)$ is generated by $\left\{\psi^{*} \alpha_{i}, \psi^{*} \beta_{i}\right\}, i \leqslant n+1 . \Psi_{\Xi}\left(\psi^{*} \alpha_{i}, \psi^{*} \beta_{j}\right)=\delta_{i j}$ and $\Psi_{\Xi}\left(\psi^{*} \alpha_{i}, \psi^{*} \alpha_{j}\right)=\Psi_{\Xi}\left(\psi^{*} B_{i}, \psi^{*} \beta_{j}\right)=0$ by (3.4) so that $\Psi_{\Xi \mid \psi^{*} H^{1}(X, Z)}$ is represented by the matrix

$$
\left[\begin{array}{cc}
0 & \operatorname{Id}_{n+1} \\
-\operatorname{Id}_{n+1} & 0
\end{array}\right] .
$$

We know that $\Psi_{\Xi}$ is represented by the matrix 


$$
\left[\begin{array}{cc}
0 & \mathrm{Id}_{g-1} \\
-\mathrm{Id}_{g-1} & 0
\end{array}\right]
$$

in any symplectic basis $B$ of $P$. Let $A$ be the matrix associated to the inclusion $\psi^{*} H^{1}(X, Z)^{\hookrightarrow} \rightarrow H^{1}(P, Z)$ in the bases $\left\{\psi^{*} \alpha_{i}, \psi^{*} \beta_{i}\right\}$ and $C$. By linear algebra,

$$
{ }^{t} A\left[\begin{array}{cc}
0 & \mathrm{Id}_{g-1} \\
-\mathrm{Id}_{g-1} & 0
\end{array}\right] A=\left[\begin{array}{cc}
0 & \mathrm{Id}_{n+1} \\
-\mathrm{Id}_{n+1} & 0
\end{array}\right]
$$

and therefore $A$ has a left inverse equal to

$$
\left[\begin{array}{cc}
0 & -\mathrm{Id}_{n+1} \\
\mathrm{Id}_{n+1} & 0
\end{array}\right]{ }^{t} A\left[\begin{array}{cc}
0 & \mathrm{Id}_{g-1} \\
-\mathrm{Id}_{g-1} & 0
\end{array}\right] .
$$

Thus $\psi^{*} H^{1}(X, Z)$ is a direct summand of $H^{1}(P, Z)$ by (1.3). Let $V^{1}$ be the complement of $\psi^{*} H^{1}(X, Z)$ and let $E^{1}$ be the subspace of $H^{1,0}(P)$ generated by $V^{1}$. It now suffices to show that $\psi^{*} H^{1,0}(X)$ is orthogonal to $E^{1}$. (Then $\hat{P}=$ $\hat{\psi}(\hat{X}) \times E^{1} / V^{1}$.)

Let $f: H^{1,0}(P) \rightarrow H^{1,0}(X)$ be the adjoint of $\psi^{*}$, i.e. such that $H_{\Xi}\left(\psi^{*} \alpha, \beta\right)$ $=H_{\Sigma}(\alpha, f \beta) \forall \alpha \in H^{1,0}(X)$. We will show that $\left.f\right|_{E^{1}}$ is identically zero, from which the conclusion follows easily.

Let $\left\{\gamma_{j}, \delta_{j}\right\}, n+1<i \leqslant g-1$, be a basis of $V^{1}$ such that $\left\{\psi^{*} \alpha_{i}, \psi^{*} \beta_{i}\right.$, $\left.\gamma_{j}, \delta_{j}\right\}$ is a symplectic basis of $\hat{P}$. We have $H_{\Sigma}\left(\alpha_{i}, f \gamma_{j}\right)=H_{\Xi}\left(\psi^{*} \alpha_{i}, \gamma_{j}\right) \in R$ because $\Psi_{\Xi}\left(\psi^{*} \alpha_{i}, \gamma_{j}\right)=0$. Similarly $H_{\Sigma}\left(\alpha_{i}, f \delta_{j}\right), H_{\Sigma}\left(\beta_{i}, f \gamma_{j}\right)$ and $H_{\Sigma}\left(\beta_{i}, f \delta_{j}\right)$ are real numbers. As $\left\{\gamma_{j}, \delta_{j}\right\}$ generate the underlying real vector space of $E^{1}, f\left(E^{1}\right) \subset$ $\left\{\alpha \in H^{1,0}(X): H_{\Sigma}(\alpha, \beta) \in R \forall \beta \in H^{1,0}(X)\right\}$. Because $H_{\Sigma}$ is nondegenerate, the only complex vector space contained in this real vector space is trivial. Thus $f\left(E^{1}\right)$ $=0$ since $\left.f\right|_{E^{1}}: E^{1} \rightarrow H^{1,0}(X)$ is a complex linear map.

Put $\hat{Y}=E^{1} / V^{1}$. Then $\hat{P}=\hat{\psi}(\hat{X}) \times \hat{Y}$ and $P \approx X \times Y$ as claimed.

(3.5) CoROllary. With the same hypotheses, if $C$ is not hyperelliptic, then $\psi$ is an isomorphism of principally polarized Abelian varieties.

Proof. It suffices to notice that the Prym variety associated to $\pi: \widetilde{C} \rightarrow$ $C$ is irreducible unless $C$ is hyperelliptic. A theorem to that effect was proved by Mumford in [M2]. Since we are going to use it again, the full statement is:

(3.6) THEOREM (MUMFORD).

(3.6.1) If $C$ is hyperelliptic, then $P$ is a hyperelliptic Jacobian or a product of two such-and hence the singular locus of $\Xi$ has dimension $g-4$ or $g-3$.

(3.6.2) If $g=3$ and $C$ is not hyperelliptic, then $P$ is a Jacobian of a curve of genus two. 
(3.6.3) If $g=4$ and $C$ is not hyperelliptic, then $P$ is a three-dimensional Jacobian. $\Xi$ is singular if and only if $P$ is a hyperelliptic Jacobian.

(3.6.4) If $C$ is not hyperelliptic and $g \geqslant 5$, then $\operatorname{dim} \Xi_{\text {sing }} \leqslant g-5$ with equality only in the following cases:

a. $C$ trigonal.

b. C-a double cover of an elliptic curve.

c. $g=5$ and there exists an even theta-characteristic $L\left({ }^{1}\right)$ with $H^{0}\left(O_{c}(L)\right) \neq 0$ and $L(D)\left({ }^{1}\right)$ even.

d. $g=6$ and there exists an odd theta-characteristic $L$ with $\operatorname{dim} H^{0}\left(O_{c}(L)\right) \geqslant 3$ and $L(D)$ even.

4. Ramified coverings. Assume that $\pi: \widetilde{C} \rightarrow C$ is a two-sheeted covering of a smooth complete algebraic curve $C$ of genus $g>0$ with $2 k$ ramification points. If $k=1$, we can construct a Prym variety associated to $\pi$ having the universal properties analogous to (2.7), (3.1) and (3.3).

Let $B=\left\{\gamma_{i}, \delta_{i}\right\}, i \leqslant g$, be a symplectic basis of $J(C)$. Then $J(\tilde{C})$ has a symplectic basis $\widetilde{B}=\left\{\gamma_{i}^{(\alpha)}, \delta_{i}^{(\alpha)}\right\}, i \leqslant g, \alpha=0,1$, such that $\left.\iota\left(\gamma_{i}^{(\alpha)}\right)=\gamma_{i}^{(1-\alpha)}\right) \iota\left(\delta_{i}^{(\alpha)}\right)$ $=\delta_{i}^{(1-\alpha)}, \pi\left(\gamma_{i}^{(\alpha)}\right)=\gamma_{i}, \pi\left(\delta_{i}^{(\alpha)}\right)=\delta_{i}$. Let $V$ be the largest subgroup of $H_{1}\left(\widetilde{C}_{1}, Z\right)$ on which $\iota$ acts as minus identity. $V$ is generated by $C=\left\{\gamma_{i}^{(0)}-\gamma_{i}^{(1)}, \delta_{i}^{(0)}-\right.$ $\left.\delta_{i}^{(1)}\right\}, i \leqslant g$. Let $E$ be the -1 eigenspace of $\iota$ in $H^{1,0}(\tilde{C})^{*}$. By RiemannHurwitz Relation, $\operatorname{dim} H^{1,0}(\widetilde{C})^{*}=2 g$, so that $\operatorname{dim} E=g$. Thus $P=E / V$ is a $g$-dimensional complex torus. The pullback of the intersection pairing on $\widetilde{C}$ to $P$ is determined by its action on $C$ :

$$
\left\langle\gamma_{i}^{(0)}-\gamma_{i}^{(1)}, \delta_{i}^{(0)}-\delta_{i}^{(1)}\right\rangle=2 \delta_{i j}
$$

and all other intersections are trivial. Thus $X=1 / 2(,,) \in H^{2}(P, Z)$ is unimodular and therefore induces a principal polarization $\Xi$ on $P$ such that $C$ is a symplectic basis. Also, $P=\operatorname{Im}\left(\operatorname{Id}_{J(\widetilde{C})}-\imath\right)$, so that $\alpha=\operatorname{Id}_{J(\widetilde{C})}-\imath: J(\widetilde{C}) \rightarrow P$ is an epimorphism.

(4.1) Proposition. If $P \neq Q$, then $\alpha \circ \tilde{t}(P) \neq \alpha \circ \tilde{t}(Q)$ unless $P$ and $Q$ are ramification points of $\pi$ or $\tilde{C}$ and $C$ are hyperelliptic.

Proof. Let $P_{0}$ and $P_{1}$ be the fixed points of $\iota$. As before, $\alpha \circ \tilde{t}(P)=$ $\alpha \circ \tilde{t}(Q)$ implies that $P+\imath Q=Q+\imath P$ by Abel's Theorem. Thus we have $P=\imath P$ and $Q=\imath Q$ or $\widetilde{C}$ is hyperelliptic with the hyperelliptic involution $j$. Since $j$ commutes with $\iota$, there is an induced involution $j_{c}$ on $C$. If $P$ is a fixed point of $j$, then so is $\iota P$ and $\pi(P)$ is a fixed point of $j_{c}$. Since $\widetilde{C}$ is of genus $2 g, j$ has $4 g+2$ fixed points and therefore $j_{c}$ has at least $2 g+1$ fixed points. Thus, by Riemann-Hurwitz Relation, $j_{c}$ is a hyperelliptic involution on $C$.

\footnotetext{
( ${ }^{1}$ We will explain these terms in $\S 5$.
} 
(4.2) Proposition. Let $X$ be an Abelian variety with the principal theta-divisor $\Sigma$. If $\varphi: \widetilde{C} \rightarrow X$ is a map such that $\varphi \circ \iota=-\varphi$ and $\varphi\left(P_{0}\right)=\varphi\left(P_{1}\right)$ $=0$, then there exists a unique homomorphism $\psi: P \rightarrow X$ such that $\psi \circ \alpha \circ$ $\tilde{t}=\varphi$.

Proof. Let $\tilde{t}$ be the standard embedding of $\widetilde{C}$ into its Jacobian with basepoint $P_{0}$. By Albanese's Theorem, there exists a homomorphism $\bar{\varphi}: J(\widetilde{C})$ $\rightarrow X$ such that $\bar{\varphi} \circ \tilde{t}=\varphi$. Since $\bar{\varphi} \circ \iota=-\bar{\varphi}$, we have $\bar{\varphi} \circ \alpha=2 \bar{\varphi}$. Restricting $\bar{\varphi}$ to $P$, we get a map $\bar{\psi}: P \rightarrow X$ such that $\bar{\psi} \circ \alpha=2 \bar{\varphi}: J(\widetilde{C}) \rightarrow X$. As in (3.1), $\alpha_{2}: J(\widetilde{C})_{2} \rightarrow P_{2}$ is onto the group of points of $P$ of order two. Therefore $P_{2}$ is contained in ker $\bar{\psi}$, and so there exists a map $\psi: P \rightarrow X$ such that $\bar{\psi}=$ $\psi \circ 2 \operatorname{Id}_{P}=2 \psi$. Thus $2 \psi \circ \alpha=2 \bar{\varphi}$ and hence $\psi \circ \alpha \circ 2 \operatorname{Id}_{\mathrm{J}(\tilde{C})}=\bar{\varphi} \circ 2 \operatorname{Id}_{\mathrm{J}(\tilde{C})}$. Since $2 \operatorname{Id}_{\mathrm{J}(\widetilde{C})}$ is an epimorphism, we have $\psi \circ \alpha=\bar{\varphi}$ and therefore $\psi \circ \alpha \circ \widetilde{t}$ $=\bar{\varphi} \circ \tilde{t}=\varphi$, as claimed.

If $\psi^{\prime}$ is another such map, we have

$$
\psi^{\prime} \circ \alpha=\bar{\varphi}=\psi \circ \alpha: J(\tilde{C}) \rightarrow X .
$$

Then $\psi^{\prime}=\psi$ as $\alpha: J(\widetilde{C}) \rightarrow P$ is onto.

(4.3) LEMMA. $[\alpha \circ \tilde{t}(\tilde{C})]=(2 /(g-1) !) \Xi(g-1)$.

ProOF.

$$
[\alpha \circ \tilde{t}(\tilde{C})]=2 \sum_{i=1}^{g}\left(\gamma_{i}^{(0)}-\gamma_{i}^{(1)}\right) \times\left(\delta_{i}^{(0)}-\delta_{i}^{(1)}\right)=\frac{2}{(g-1) !} \Xi(g-1)
$$

since $C$ is a symplectic basis of $P$.

(4.4) THEOREM. With the hypotheses of Proposition (4.2) if $\varphi(\tilde{C})$ represents the homology class $(2 / n !) \Sigma^{(n)}$ in $H_{2}(X, Z)$, then $X$ is isomorphic to a factor of $P$.

Proof. As in (3.3),

$$
\psi_{*}\left(\frac{1}{(g-1) !} \Xi(g-1)\right)=\frac{1}{n !} \Sigma^{(n)} \text {. }
$$

Therefore $H_{\Xi}\left(\psi^{*} \alpha, \psi^{*} \beta\right)=H_{\Sigma}(\alpha, \beta) \forall \alpha, \beta \in H^{1,0}(X)$ and the proof is the same as (3.3).

When $k \geqslant 2$, the polarization of $P$ induced by the intersection pairing on $\widetilde{C}$ is neither principal nor a multiple of one. With some additional information we can, however, construct an Abelian variety isogenous to $P$ with a principal theta-divisor $\Xi$ having the desired properties.

Assume that $\pi: \widetilde{C} \rightarrow C$ is ramified at $\left\{P_{j}^{\alpha}\right\}, 0 \leqslant j<k, \alpha=0,1$. Then $J(\widetilde{C})$ has a symplectic basis $\widetilde{B}=\left\{\gamma_{i}^{(\alpha)}, \delta_{i}^{(\alpha)}, \sigma_{j}, \rho_{j}\right\}, i \leqslant g, \alpha=0,1, j<k$, such that $\iota\left(\gamma_{i}^{(\alpha)}\right)=\gamma_{i}^{(1-\alpha)}, \iota\left(\delta_{i}^{(\alpha)}\right)=\delta_{i}^{(1-\alpha)}, \iota\left(\sigma_{j}\right)=-\sigma_{j}, \iota\left(\rho_{j}\right)=-\rho_{j}, \pi\left(\gamma_{i}^{(\alpha)}\right)$ 
$=\gamma_{i}, \pi\left(\delta_{i}^{(\alpha)}\right)=\delta_{j}$ and $\pi\left(\sigma_{j}\right)$ and $\pi\left(\rho_{j}\right)$ are homologous to zero on $C . \sigma_{j}$ is the cycle passing through $P_{j}^{0}$ and $P_{j}^{1}$ for $j>0$ and $\rho_{j}$ is the complementary cycle (see figure).

Let $E$ be the -1 eigenspace of $\iota$ in $H^{1,0}(\widetilde{C})^{*}$. By Riemann-Hurwitz Relation, $\operatorname{dim} E=g+k-1$. Let $V$ be the largest subgroup of $H_{1}(\widetilde{C}, Z)$ on which $\iota$ acts as minus identity. $V$ is the lattice in $E$ generated by $\left\{\gamma_{i}^{(0)}-\gamma_{i}^{(1)}\right.$, $\left.\delta_{i}^{(0)}-\delta_{i}^{(1)}, \sigma_{j}, \rho_{j}\right\}, i \leqslant g, j<k$. Let $V_{0} \subset V$ be the subgroup generated by $C_{0}=\left\{\gamma_{i}^{(0)}-\gamma_{i}^{(1)}, \delta_{i}^{(0)}-\delta_{i}^{(1)}, \sigma_{j}, 2 \rho_{j}\right\}$. Then $P_{0}=E / V_{0}$ has a principal polarization $\Xi$ induced by the principal polarization $\widetilde{\Theta}$ of $J(\widetilde{C})$ such that $C_{0}$ is a symplectic basis of $P_{0}$. As we have already remarked, $P=E / V$ does not have such a polarization.

(4.5) Definition. $P_{0}$ with the principal polarization $\Xi$ is called the Prym variety associated to the two-sheeted covering $\pi: \widetilde{C} \rightarrow C$ with ramification points $\left\{P_{j}^{\alpha}\right\}, 0 \leqslant j<k, \alpha=0,1$, paired by the index $j$.

$P_{0}$ depends on the pairing of ramification points.

Since $V_{0} \subset V$, we have an isogeny $\beta: P_{0} \rightarrow P$. Let $\alpha=\operatorname{Id}_{\mathrm{J}(\widetilde{C})}-\imath$ : $J(\tilde{C}) \rightarrow J(\widetilde{C})$. It is easily shown that $\operatorname{Im} \alpha=P$. Let $\alpha_{\#}: H_{1}(\widetilde{C}, Z) \rightarrow V$ be the induced homomorphism of fundamental groups. Then $\operatorname{Im} \alpha_{\#} \subset V_{0}$ and so there exists a homomorphism $\tilde{\alpha}: J(\widetilde{C}) \rightarrow P_{0}$ such that $\alpha=\beta \circ \widetilde{\alpha}$. We can construct $\tilde{\alpha}$ explicitly:

Let $P \in \widetilde{C}$ and let $D$ be a path on $\widetilde{C}$ such that

$$
D(0)=P, \quad D(1 / 2)=P_{0}^{0}, \quad \text { and } \quad D(1-t)=i D(t) .
$$

Note that if $D^{\prime}$ is another path on $\widetilde{C}$ satisfying (*), then $D-D^{\prime} \in \operatorname{Im} \alpha_{\#} \subset V_{0}$. Therefore $\int_{D}-\epsilon E$ and $\bar{\alpha}(P)=\int_{D}-\bmod V_{0}$ is well defined. $\tilde{\alpha}$ is obtained from $\bar{\alpha}: \widetilde{C} \rightarrow P$ by extension to divisors on $\widetilde{C}$. We have $\tilde{\alpha} \circ \tilde{t}=\bar{\alpha}: \widetilde{C} \rightarrow P$. Note that $\bar{\alpha}\left(P_{0}^{0}\right)=\bar{\alpha}\left(P_{0}^{1}\right)=0$ and for $j>0, \bar{\alpha}\left(P_{j}^{0}\right)=\bar{\alpha}\left(P_{j}^{1}\right)=\rho_{j}$ is a point of order two of $P_{0}$.

(4.6) Proposition. If $P \neq Q$, then $\alpha(P) \neq \alpha(Q)$ unless $P=P_{j}^{0}$ and $Q=$ $P_{j}^{1}$ for some $0 \leqslant j<k$ or, if $k=2, \widetilde{C}$ and $C$ are hyperelliptic.

Proof. $\bar{\alpha}(P)=\bar{\alpha}(Q)$ implies $P-\imath P=Q-\iota Q$. Thus either $P$ and $Q$ are a pair of ramification points with the same index $j$ or $\widetilde{C}$ has a hyperelliptic involution $\tilde{j}$ with $4 g+2 k$ fixed points. Again as in (2.8) and (4.1) $\tilde{j}$ commutes with $\iota$ and therefore induces an involution $j_{c}$ on $C$. If $P$ is a fixed point of $\tilde{j}$, then so is $\iota P$ and $\pi(P)$ is a fixed point of $j_{c}$. Thus $j_{c}$ has at least $2 g+k$ fixed points. Since no involution on $C$ has more than $2 g+2$ fixed points, and since an involution with $2 g+2$ fixed points is hyperelliptic, the conclusion follows. 
(4.7) Proposition. Let $X$ be an Abelian variety with principal polarization $\Sigma$. If $\varphi: \widetilde{C} \rightarrow X$ is a map such that $\varphi \circ \iota=-\varphi, \varphi\left(P_{0}^{0}\right)=\varphi\left(P_{0}^{1}\right)=0$ and $\varphi\left(P_{j}^{g}\right)=\varphi\left(P_{j}^{1}\right)$, then there exists a unique $\psi: P \rightarrow X$ such that $\psi \circ \bar{\alpha}=\varphi$.

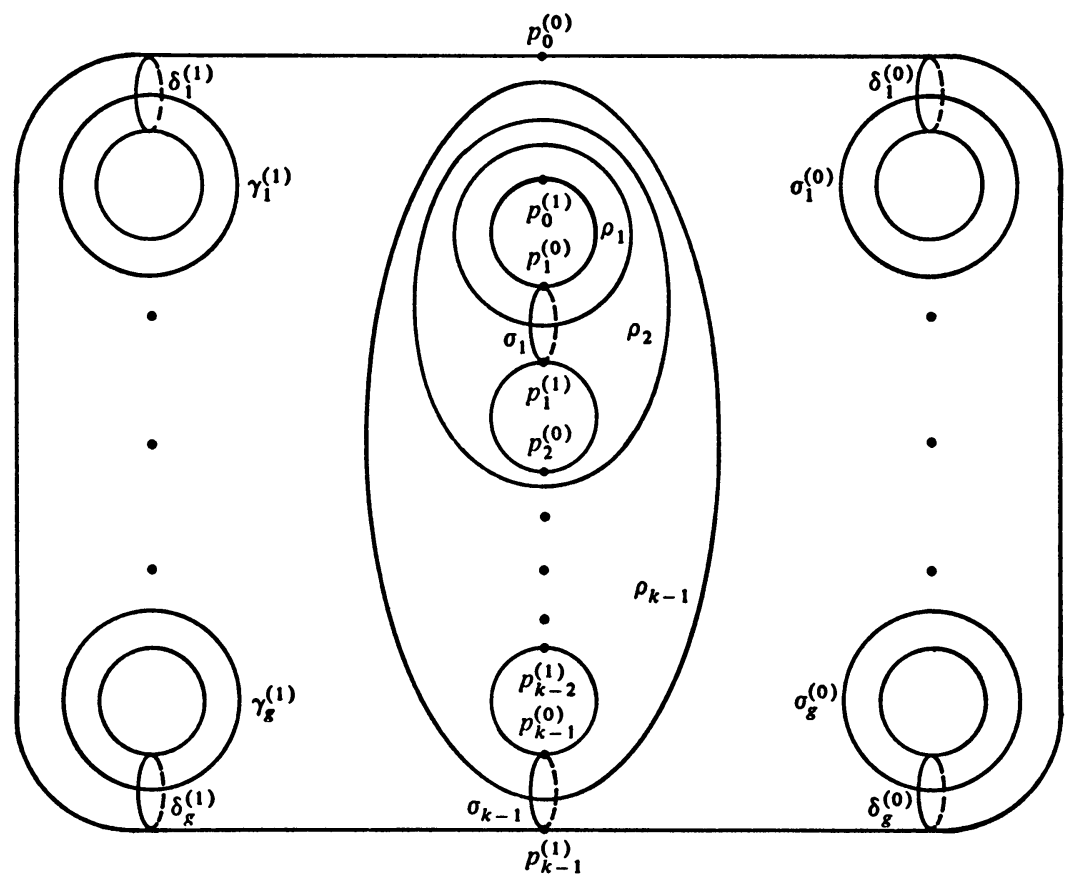

Proof. Let $\bar{\varphi}: J(\tilde{C}) \rightarrow X$ be the homomorphism induced by $\varphi$. Then $\bar{\varphi} \circ \tilde{t}=\varphi$, where $\tilde{t}: \widetilde{C} \rightarrow J(\tilde{C})$ has the basepoint $P_{0}^{0}$ and $\bar{\varphi} \circ \iota=-\bar{\varphi}$ so that $\bar{\varphi} \circ \alpha=2 \bar{\varphi}$. Put $\bar{\psi}=\left.\bar{\varphi}\right|_{P}$. Since $P=\operatorname{Im} \alpha$, we have $\bar{\psi} \circ \alpha=\bar{\varphi} \circ \alpha=2 \bar{\varphi}$. Let $\tilde{\psi}=\bar{\psi} \circ \beta: P_{0} \rightarrow X$. Then $\tilde{\psi} \circ \tilde{\alpha}=\bar{\psi} \circ \beta \circ \tilde{\alpha}=\bar{\psi} \circ \alpha=2 \bar{\varphi}$, so that we have a commutative diagram:

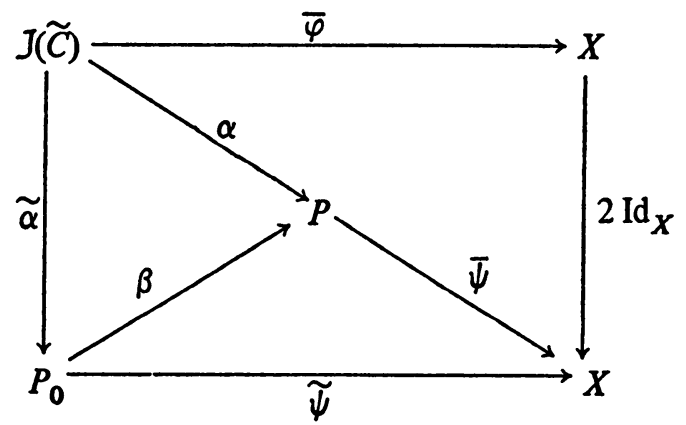

Again, as in (3.1) and (4.2) we need a map $\psi: P_{0} \rightarrow X$ such that $\tilde{\psi}=2 \psi$. $\left(P_{0}\right)_{2}$ is generated by $\widetilde{\alpha}\left(J(\widetilde{C})_{2}\right) \cup\left\{\rho_{j}\right\}, j<k$. Therefore $\beta\left(\left(P_{0}\right)_{2}\right)$ is generated by $\beta \circ \tilde{\alpha}\left(J(\widetilde{C})_{2}\right) \cup\left\{\beta\left(\rho_{j}\right)\right\}$. But $\beta\left(\rho_{j}\right)=0$ for all $j$, so that $\beta\left(\left(P_{0}\right)_{2}\right)=$ $\beta \circ \tilde{\alpha}\left(J(\widetilde{C})_{2}\right)=\alpha\left(J(\widetilde{C})_{2}\right)$. Thus $\widetilde{\psi}\left(\left(P_{0}\right)_{2}\right)=\bar{\psi} \circ \beta\left(\left(P_{0}\right)_{2}\right)=\bar{\psi} \circ \alpha\left(J(\widetilde{C})_{2}\right)=$ 
$2 \bar{\varphi}\left(J(\widetilde{C})_{2}\right)=0$ since every element of $\bar{\varphi}\left(J(\widetilde{C})_{2}\right)$ is of order two in $X$.

Thus $\tilde{\psi}$ factors through $2 \mathrm{Id}_{P}$, giving a homomorphism $\psi: P \rightarrow X$ such that $\tilde{\psi}=2 \psi$ and therefore $\psi \circ \tilde{\alpha}=\bar{\varphi}$, as claimed.

If $\psi^{\prime}$ is another such map, then we have $\psi^{\prime} \circ \tilde{\alpha}=\bar{\varphi}=\psi \circ \tilde{\alpha}: J(\tilde{C}) \rightarrow X$ and therefore $\psi^{\prime}=\psi$ since $\tilde{\alpha}: J(\widetilde{C}) \rightarrow P_{0}$ is an epimorphism.

(4.8) LEMMA. $[\bar{\alpha}(\tilde{C})]=(2 /(g+k-2) !) \Xi^{(g+k-2)}$.

Proof. $[\tilde{t}(\tilde{C})]=\Sigma_{i, \alpha} \gamma_{i}^{(\alpha)} \times \delta_{i}^{(\alpha)}+\Sigma_{j} \sigma_{j} \times \rho_{j} \in H_{2}(J(\tilde{C}), Z)$. Therefore

$$
\begin{aligned}
{[\tilde{\alpha}(\tilde{C})] } & =[\tilde{\alpha} \circ \tilde{t}(\tilde{C})] \\
& =\sum_{i, \alpha} \tilde{\alpha}\left(\gamma_{i}^{(\alpha)}\right) \times \tilde{\alpha}\left(\delta_{i}^{(\alpha)}\right)+\sum_{j} \tilde{\alpha}\left(\sigma_{j}\right) \times \tilde{\alpha}\left(\rho_{j}\right) \\
& =2 \sum_{i=1}^{g}\left(\gamma_{i}^{(0)}-\gamma_{i}^{(1)}\right) \times\left(\delta_{i}^{(0)}-\delta_{i}^{(1)}\right)+\sum_{j=1}^{k-1} 2 \sigma_{j} \times 2 \rho_{j} \\
& =2\left(\sum_{i=1}^{g}\left(\gamma_{i}^{(0)}-\gamma_{i}^{(1)}\right) \times\left(\delta_{i}^{(0)}-\delta_{i}^{(1)}\right)+\sum_{j=1}^{k-1} \sigma_{j} \times 2 \rho_{j}\right) \\
& =\frac{2}{(g+k-2) !} \Xi(g+k-2)
\end{aligned}
$$

since $C_{0}$ is a symplectic basis of $P_{0}$.

(4.9) THEOREM. With the hypothesis of Proposition (4.7), if $\varphi(\widetilde{C})$ represents the homology class $(2 / n !) \Sigma^{(n)}$ in $H_{2}(X, Z)$, then $X$ is isomorphic to a factor of $P_{0}$.

In view of Lemma (4.8), the proof is identical to (3.3) and (4.4).

(4.10) Corollary. If $P_{0}$ is irreducible, then $P_{0} \approx X$. If $\operatorname{dim} P_{0}=$ $\operatorname{dim} X$, then $P_{0} \approx X$.

5. Curves of genus five and plane quintics. The main references for this section are [M2], [M3] and [AM].

It is well known that unramified two-sheeted coverings $\pi: \widetilde{C} \rightarrow C$ correspond to the points of $J(C)_{2}$, i.e. divisor classes $D$ on $C$ such that $2 D=0$. Let $K_{C}$ be the canonical divisor on $C$. Consider divisor classes $U$ such that $2 U=K_{C}$. These "half-canonical" classes, called theta-characteristics, form a principal homogeneous space over $\mathrm{J}(C)_{2}$. (If $U$ is a theta-characteristic and $D$ is a half-period, then $2(U+D)=K_{C}$, so that $U+D$ is a theta-characteristic and if $U_{1}, U_{2}$ are theta-characteristics, then $2\left(U_{1}-U_{2}\right)=0$, so that $U_{1}-U_{2}$ is a half-period.)

Let $U_{0}$ be a fixed theta-characteristic. $U_{0}$ gives rise to the map $\epsilon: J(\widetilde{C})_{2}$ $\rightarrow Z_{2}$ given by 


$$
\epsilon(D)=\operatorname{dim} H^{0}\left(O_{C}\left(U_{0}+D\right)\right)(\bmod 2) .
$$

Let $C$ be a smooth plane curve of degree five. The canonical class $K_{C}$ is cut out on $C$ by the linear series of plane quadrics. Therefore the series $O_{C}(1)$, cut out on $C$ by lines in the plane is a distinguished theta-characteristic. In the notation of Andreotti and Mayer [AM] $O_{C}(1)$ is a $g_{5}^{2} . \quad\left(g_{n}^{r}\right.$ is a divisor class of degree $n$ and dimension $r+1$.) The associated parity map $\epsilon$ decomposes $J(C)_{2}$ into two subsets according to whether $\operatorname{dim} H^{0}\left(O_{C}(1) \otimes O_{C}(D)\right)$ is even or odd. It is known (compare [FR]) that there are $2^{g-1}\left(2^{g}+1\right)$ even and $2^{g-1}\left(2^{g}-1\right)$ odd half-periods.

Let $\pi: \widetilde{C} \rightarrow C$ be the unramified two-sheeted covering corresponding to the even half-period $D$. Then $\pi$ satisfies one of the hypotheses of Mumford's Theorem (3.6.4.d) and therefore the singular locus of the theta-divisor $\Xi$ on the associated Prym variety $P$ is one dimensional. In fact, the singular points of $\Xi$ correspond to the divisors of the form $\pi^{*}\left(g_{5}^{2}\right)+(P-\imath P)$ on $\widetilde{C}$ (compare [M2]).

(5.1) Proposition. The singular locus of $\Xi$ is isomorphic to $\widetilde{C}$.

Proof. In view of Proposition (2.7), we only have to prove that a nonsingular plane quintic is not hyperelliptic. Clifford's Theorem states that if there is a $g_{n}^{r}$ on $C$, then $2 r \leqslant n$ and $2 r=n$ only if $g_{n}^{r}$ is the canonical divisor, empty divisor or $C$ is hyperelliptic and $g_{n}^{r}$ is a multiple of the $g_{2}^{1}$ on $C$. Assume that $C$ is a smooth plane quintic (and thus carrying a $g_{5}^{2}$ ) which is hyperelliptic (carrying a $g_{2}^{1}$ ). Let $D_{1}$ be a divisor in $g_{5}^{2}$ and $D_{2}$ in $g_{2}^{1}$. Then an elementary calculation shows that $\left|D_{1}+D_{2}\right|=g_{7}^{5}$, which contradicts Clifford's Theorem.

Let $\Gamma$ be a smooth complete nonhyperelliptic curve of genus five. Let $J=J(\Gamma)$ be the Jacobi variety of $\Gamma$ with the principal theta-divisor $\Sigma$. $\Sigma$ has one-dimensional singular locus $\widetilde{C}$ symmetric in $J$ (i.e. if $x \in \widetilde{C}$, then so does $-x$ ) because the Riemann's theta function is even in each variable. Thus we have an involution $\iota=-\operatorname{Id}_{\mathrm{J} \mid \widetilde{C}}$ on $\widetilde{C}$. Let $C$ be the quotient curve. We want to examine the two-sheeted covering $\pi: \widetilde{C} \rightarrow C$.

(5.2) LemMA. There are no triple points on $\Sigma$.

Proof. By Riemann's Singularity Theorem $\Sigma$ has a triple point if and only if $\Gamma$ carries $g_{4}^{2}$. By Clifford's Theorem, a nonhyperelliptic curve of genus five does not carry a $g_{4}^{2}$.

Let $x \in \widetilde{C}$ and let $Q_{x}$ be the tangent cone to $\Sigma$ at ${ }^{8 \mathrm{PI}} Q_{x}$ translated to the origin gives rise to a quadratic hypersurface, also denoted by $Q_{x}$, in $P\left(T_{\mathrm{J}, 0}\right)=$ $P\left(H^{0}\left(\Omega_{\Gamma}^{1}\right)^{*}\right) . Q_{x}$ is singular because $x$ is not an isolated singularity of $\Sigma$ and therefore the tangent cone has $T_{\widetilde{C}, x}$ as a vertex. It is shown by Andreotti and Mayer [AM] that $Q_{x}$ contains the canonical image of $\Gamma$ in $P C_{4}=P\left(H^{0}\left(\Omega_{\Gamma}^{1}\right)^{*}\right)$. 
As quadrics of rank one and two are reducible and the canonical image of $\Gamma$ is contained in no hypersurface in $P C_{4}$, we have rk $Q_{x} \geqslant 3$. On the other hand, singular quadrics in $P_{4}$ are of rank at most four, so that rk $Q_{x}$ is either three or four.

By Noether's Theorem quadrics containing the canonical image of $\Gamma$ form a two-dimensional projective system $S_{\Gamma}$. If $\Gamma$ is not trigonal (i.e. it does not carry a $g_{3}^{1}$ ), there are nonsingular quadrics in $S_{\Gamma}$ and the canonical image of $\Gamma$ is a complete intersection in $P C_{4}$. Let $Q_{0}, Q_{1}, Q_{2}$ be such elements of $S_{\Gamma}$ that $Q_{0} \cap Q_{1} \cap Q_{2}=\Gamma$. Then every quadric $Q$ containing $\Gamma$ can be written as $Q=$ $t_{0} Q_{0}+t_{1} Q_{1}+t_{2} Q_{2}$ for some $\left(t_{0}, t_{1}, t_{2}\right) \in P C_{2}$. Therefore $Q \in S_{\Gamma}$ for $\Gamma$ nontrigonal is singular if and only if

$$
f\left(t_{0}, t_{1}, t_{2}\right)=\operatorname{det}\left|t_{0} Q_{0}+t_{1} Q_{1}+t_{2} Q_{2}\right|=0 .
$$

Assigning to every point $x \in \widetilde{C}$ the tangent cone $Q_{x}$ gives therefore a map from $\widetilde{C}$ to the fifth degree projective plane curve $f\left(t_{0}, t_{1}, t_{2}\right)=0$. Since the Riemann's theta function is even, $x$ and $-x$ in $\widetilde{C}$ are mapped to the same point of the plane quintic. On the other hand, every quadric $Q \in S_{\Gamma}$ gives rise to a pair of points $(x,-x)$ of $\widetilde{C}$ such that $Q=Q_{x}$ and $x=-x$ if and only if rk $Q=$ 3 (see [AM] for the proof). Therefore $C=\widetilde{C} / \iota$ is the plane quintic $f\left(t_{0}, t_{1}, t_{2}\right)$ $=0$ and $\pi: \widetilde{C} \rightarrow C$ is the map described above.

If $\Gamma$ is trigonal, then $\widetilde{C}$ consists of two intersecting copies of $\Gamma \subset J$ interchanged by $i=-\mathrm{Id}_{\mathrm{J} \mid \widetilde{C}} \cdot S_{\Gamma}$ contains no nonsingular quadrics and $C$ consists of quadrics whose vertex meets the canonical image of $\Gamma$. It can be shown (compare [E]) that in this case $C$ is a plane quintic with one double point birationally equivalent to $\Gamma$.

If $\Gamma$ is not trigonal, the canonical image of $\Gamma$ does not meet the vertex of any quadric $Q \in S_{\Gamma}$ and the singular locus $\widetilde{C}$ of $\Sigma$ is irreducible (viz. [AM]).

(5.3) Proposition. The following conditions are equivalent:

(a) $\widetilde{C}$ is smooth.

(b) $\widetilde{C}$ misses $J_{2}$ (i.e. there are no vanishing even theta-nulls on $\Gamma$ ).

(c) $\pi: \widetilde{C} \rightarrow C$ is unramified.

(d) $C$ is smooth.

(e) The quadrics in $C$ are all of rank four.

Proof. $\mathrm{b} \Longleftrightarrow \mathrm{c}: x$ is a ramification point if and only if $x=-x$. c $\Longleftrightarrow \mathrm{e}: x=-x$ if and only if $Q_{x}$ is of rank three.

$\mathrm{a} \Longleftrightarrow \mathrm{e}: x$ is a singular point of $\widetilde{C}$ if and only if $T \widetilde{C}, x \geqslant 2$ if and only if the vertex of $Q_{x}$ is at least one dimensional if and only if $\operatorname{rk} Q_{x}<4$.

Finally, we shall prove $\mathrm{d} \Longleftrightarrow \mathrm{e}$.

If $C$ is smooth, then $\Gamma$ is not trigonal and there exist quadrics $Q_{0}, Q_{1}, Q_{2}$ 
in $S_{\Gamma}$ such that $\Gamma=Q_{0} \cap Q_{1} \cap Q_{2}$ and $C$ is the zero locus of $f\left(t_{0}, t_{1}, t_{2}\right)=$ $\operatorname{det}\left|\Sigma_{i=0}^{2} t_{i} Q_{i}\right|$. Choose $Q_{0}$ singular. Then $(1,0,0) \in C$ and we shall prove that it is a singular point of $C$ if and only if $\mathrm{rk} Q_{0}=3$.

Let $Q_{i}$ be given by the symmetric matrix $\left(q_{k j}^{i}\right)$. Let $M_{k j}$ be the $4 \times 4$ minor complementing the $(k, j)$ entry of the matrix $\Sigma_{i=0}^{2} t_{i} Q_{i}$. Then

$$
\frac{\partial f}{\partial t_{i}}\left(t_{0}, t_{1}, t_{2}\right)=\sum_{k, j=1}^{5}(-1)^{k+j} q_{k j}^{i} \operatorname{det} M_{k j}\left(t_{0}, t_{1}, t_{2}\right) .
$$

Note that $M_{k j}(1,0,0)$ is the minor complementing the $(k, j)$ entry in the matrix $Q_{0}=\left(q_{k j}^{0}\right)$.

If rk $Q_{0}=3$, then every $4 \times 4$ minor of $Q_{0}$ is singular. Therefore

$$
\frac{\partial f}{\partial t_{i}}(1,0,0)=\sum_{k, j=1}^{5}(-1)^{k+j} q_{k j}^{0} \operatorname{det} M_{k j}(1,0,0)=0
$$

so that $(1,0,0)$ is a singular point of $C$.

If rk $Q_{0}=4$, choose $Q_{1} \in S_{\Gamma}-C$ such that $\operatorname{det}\left|Q_{0}+\lambda Q_{1}\right|$ is a square free polynomial in $C[\lambda]$. Such a quadric always exists-otherwise every line through $(1,0,0)$ would have a point of second order contact with $C$.

Then (compare [HP]) $Q_{0}$ and $Q_{1}$ can be simultaneously diagonalized. Thus $Q_{0}=\operatorname{diag}(1,1,1,1,0)$ and $Q_{1}=\operatorname{diag}\left(\alpha_{1}, \ldots, \alpha_{5}\right)$ with $\alpha_{i} \neq 0$.

The matrix $Q_{0}$ has only one nonsingular $4 \times 4$ minor, namely $M_{55}(1,0,0)$ and $\operatorname{det} M_{55}(1,0,0)=1$. Thus

$$
\begin{aligned}
\frac{\partial f}{\partial t_{i}}(1,0,0) & =\sum_{k, j}(-1)^{k+j} q_{k j}^{1} \operatorname{det} M_{k j}(1,0,0) \\
& =q_{55}^{1} \operatorname{det} M_{55}(1,0,0)=\alpha_{5} \neq 0
\end{aligned}
$$

so that $(1,0,0)$ is a smooth point of $C$.

Let us examine the singularities of $C$. Again, $Q_{0}$ is a quadric of rank at most four passing through $\Gamma$ (i.e. containing the canonical image of $\Gamma$ ) and $Q_{1}$, $Q_{2}$ are elements of $S_{\Gamma}$ of maximal rank (we assume that $\Gamma$ is not trigonal).

(5.4) Lemma. If $\mathrm{rk} Q_{0}=3$, then $(1,0,0)$ is a node on $C$.

Proof. Choose projective coordinates in $P C_{4}$ so that $Q_{0}$ is represented by the matrix $\operatorname{diag}(0,0,1,1,1)$. The line $x_{2}=x_{3}=x_{4}=0$ is the vertex of $Q_{0}$. We have 


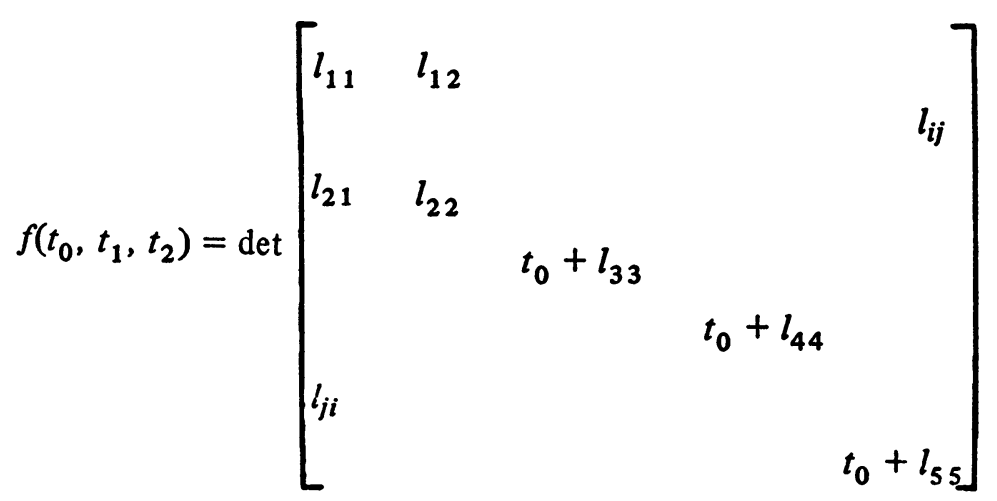

where $l_{i j}=l_{i j}\left(t_{1}, t_{2}\right)$ are linear forms. Thus

$$
f\left(t_{0}, t_{1}, t_{2}\right)=\operatorname{det}\left[\begin{array}{ll}
l_{11} & l_{12} \\
l_{21} & l_{22}
\end{array}\right]\left(t_{0}+l_{33}\right)\left(t_{0}+l_{44}\right)\left(t_{0}+l_{5 \xi}\right)+\cdots
$$

(forms not involving $t_{0}^{3}$ ).

Therefore in the neighborhood of $(1,0,0)$

$$
f\left(1, t_{1}, t_{2}\right)=\operatorname{det}\left[\begin{array}{ll}
l_{11} & l_{12} \\
l_{21} & l_{22}
\end{array}\right]+\cdots \text { (terms of higher degree). }
$$

Hence

$$
\operatorname{det}\left[\begin{array}{ll}
l_{11} & l_{12} \\
l_{21} & l_{22}
\end{array}\right]=0
$$

is the tangent cone to $C$ at $(1,0,0)$ provided it is not identically zero.

Assume

$$
\operatorname{det}\left[\begin{array}{ll}
l_{11} & l_{12} \\
l_{21} & l_{22}
\end{array}\right]=0
$$

Changing the projective coordinates in $P C_{2}=S_{\Gamma}$ if necessary, we may assume that $l_{11}=t_{1}$. Then we have

$$
\operatorname{det}\left[\begin{array}{cc}
t_{1} & \alpha t_{1}+\beta t_{2} \\
\alpha t_{1}+\beta t_{2} & \gamma t_{1}+\delta t_{2}
\end{array}\right]=0,
$$

which implies $\beta=\delta=0$ and $\gamma=\alpha^{2}$. If $\alpha=0$, we have 


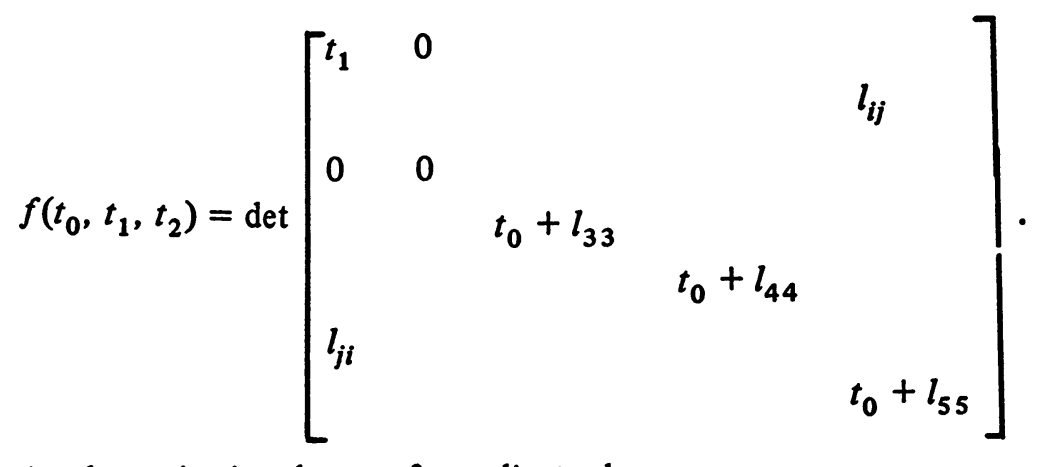

Otherwise the projective change of coordinates by

$$
\left[\begin{array}{rrrrr}
1 & -\alpha & 0 & 0 & 0 \\
0 & 1 & 0 & 0 & 0 \\
0 & 0 & 1 & 0 & 0 \\
0 & 0 & 0 & 1 & 0 \\
0 & 0 & 0 & 0 & 1
\end{array}\right]
$$

brings $f$ to this form.

$(0,1,0,0,0) \in P C_{4}$ lies in every quadric containing $\Gamma$, hence belongs to $\Gamma$. But it is contained in the vertex of $Q_{0}$, which cannot happen if $\Gamma$ is not trigonal.

Therefore $(1,0,0) \in P C_{2}$ is a double point of $C$ and it is a cusp if and only if

$$
\operatorname{det}\left[\begin{array}{ll}
l_{11} & l_{12} \\
l_{21} & l_{22}
\end{array}\right]=l^{2}\left(t_{1}, t_{2}\right)
$$

Changing the projective coordinates if necessary, we may assume that $l_{12}=l_{21}$ $=0, l_{11}=t_{1}, l_{22}=\alpha t_{1}$.

Then

$$
f\left(t_{0}, t_{1}, t_{2}\right)=\operatorname{det}\left[\begin{array}{ccccc}
t_{1} & 0 & & \\
0 & \alpha t_{1} & & & \\
& & t_{0}+l_{33} & & \\
l_{j i} & & & t_{0}+l_{44} & \\
& & & & t_{0}+l_{55}
\end{array}\right] \text {. }
$$

Every quadric of the form $t_{0} Q_{0}+t_{2} Q_{2}$ contains the line $x_{2}=x_{3}=x_{4}=0$ in 
$P C_{4}$. Thus $\Gamma=Q_{0} \cap Q_{1} \cap Q_{2}$ meets this line and therefore meets the singular locus of $Q_{0}$, which cannot happen unless $\Gamma$ is trigonal.

If $C$ is a plane quintic with double points and $\pi: \widetilde{C} \rightarrow C$ is a two-sheeted covering, let us consider the normalized two-sheeted covering $\bar{\pi}: \widetilde{C} \rightarrow \bar{C}$.

(5.5) Corollary. The Prym variety $P$ associated to $\bar{\pi}$ is five dimensional if and only if $\bar{\pi}$ is ramified at all the preimages in $\bar{C}$ of nodes in $C$, unless $\Gamma$ is trigonal.

Proof. Assume $\Gamma$ is not trigonal. Then $\widetilde{C}$ and $\overline{\widetilde{C}}$ are irreducible. Let $k$ be the number of nodes of $C$. Then genus of $\bar{C}$ equals $6-k$ and $\overline{\widetilde{C}}$ is of genus $2(6-k)-1+l$ where $2 l$ is the ramification index of $\bar{\pi}$. Thus $\operatorname{dim} P=$ $(11-2 k+l)-(6-k)=5-k+l$ and $\operatorname{dim} P=5$ if and only if $k=l$. Since the only possible ramifications are above the $2 k$ preimages of nodes of $C$ by (5.3), all of these points must be branchpoints of $\bar{\pi}$.

Since the branchpoints of $\bar{\pi}$ (henceforth denoted by $\pi$ ) are paired by the nodes of $C$, we can construct the isogenous variety $P_{0}$. We have the main theorem of this section.

(5.6) THEOREM. For every smooth complete nonhyperelliptic curve $\Gamma$ of genus five, there exists a two-sheeted covering $\pi: \widetilde{C} \rightarrow C$ (possibly ramified) of a plane quintic $C$ such that the Jacobi variety $J(\Gamma)$ and the Prym variety $P$ (or $P_{0}$ ) associated to $\pi$ are isomorphic Abelian varieties.

Proof. We have constructed $\pi: \widetilde{C} \rightarrow C$. Let $\varphi^{\prime}: \widetilde{C} \rightarrow J(=J(\Gamma))$ be the inclusion map. $\varphi^{\prime}(\iota P)=-\varphi^{\prime}(P)$ and $\varphi^{\prime}(\iota P)=\varphi^{\prime}(P)$ if and only if $P$ is a ramification point of $\pi$. Choose a pair $\left(P_{0}^{0}, P_{0}^{1}\right)$ of ramification points such that $\varphi^{\prime}\left(P_{0}^{0}\right)=$ $\varphi^{\prime}\left(P_{0}^{1}\right)$ and let $\varphi(P)=\varphi^{\prime}(P)-\varphi^{\prime}\left(P_{0}^{0}\right)$. Again, $\varphi((P)=-\varphi(P)$ and $\varphi(L P)=\varphi(P)$ if and only if $P$ is a ramification point of $\pi$. Moreover, $\varphi\left(P_{j}^{0}\right)=\varphi\left(P_{j}^{1}\right)$ for every $j, 0$ $\leqslant j<k$. Therefore by Propositions (3.1), (4.2) and (4.7) there exists a map $\psi$ : $P \rightarrow J\left(\psi: P_{0} \rightarrow J\right.$ if $\pi$ is ramified $)$ such that $\varphi=\psi \circ \alpha \circ \tilde{t}(=\psi \circ \tilde{\alpha} \circ \tilde{t})$.

Let $E_{d}^{l}=\left\{g_{d}^{r}\right.$ on $\left.\Gamma: r \geqslant l-1\right\} . E_{d}^{l} \subset J$ and is an integral cycle. The Castelnuovo's Formula (compare $[\mathrm{K}]$ ) tells us that if codimension of this cycle is at least $l(l-d+g-1)$, then this cycle represents the homology class

$$
\prod_{0<i<l} \frac{i !}{(l-d+g-1+i) !} \Sigma^{l(l-d+g-1)} .
$$

By Riemann's Singularity Theorem, the singular locus of $\Sigma$ equals $E_{4}^{2}$. Thus $\widetilde{C}$ (strictly, $\varphi(\widetilde{C})$ ) represents the homology class

$$
\frac{1}{2 !} \cdot \frac{1}{3 !} \Sigma^{(4)}=\frac{2}{4 !} \Sigma^{(4)}
$$


since codimension of $\widetilde{C}$ in $J$ equals $4=2(2-4+5-1)$. Therefore $J$ is a factor of $P$ (or $P_{0}$ ) by Theorems (3.3), (4.4) and (4.9) and is isomorphic to $P$ by Corollary (3.4) (or to $P_{0}$, if $\pi$ is ramified, by Corollary (4.10)).

Proposition (5.1) implies uniqueness of $\pi$, if $\pi$ is unramified.

Assume now $\widetilde{C}$ is reducible. Then $\Gamma$ is trigonal and isomorphic to $\bar{C}$. Thus $J(\widetilde{\widetilde{C}})=J(\bar{C}) \times J(\bar{C})$ with $\iota$ interchanging factors. Then

$$
\begin{gathered}
P=\{(x, y) \in J(\widetilde{\widetilde{C}}):(x, y)=-(y, x)\} \\
=\{(x, y) \in J(\widetilde{\widetilde{C}}): x=-y\} \approx J(\widetilde{C})=J . \\
\text { REFERENCES }
\end{gathered}
$$

[AM] A. Andreotti and A. Mayer, On period relations for abelian integrals on algebraic curves, Ann. Scuola Norm. Sup. Pisa (3) 21 (1967), 189-238. MR 36 \#3792.

[CG] H. Clemens and P. Griffiths, The intermediate Jacobian of the cubic threefold, Ann. of Math. (2) 95 (1972).

[E] F. Enriques, Teoria geometrica delle equazioni.

[F] H. Farkas, Automorphisms of compact Riemann surfaces, Ann. of Math. Studies, no. 79, Princeton Univ. Press, Princeton, N. J.

[FR] H. Rauch and H. Farkas, Theta functions with applications to Riemann surfaces, Williams and Wilkins, Baltimore, Md., 1974. MR 50 \#4595.

[HP] W. V. D. Hodge and D. Pedoe, Methods of algebraic geometry. Vols. I, II, III, Cambridge Univ. Press, New York, 1947, 1952, 1954. MR 10, 396; 13, 972; 15, 893.

[K] G. Kempf, Schubert manifolds with an application to algebraic curves, Stichting Math. Centrum, Amsterdam, 1971.

[M1] D. Mumford, A belian varieties, Tata Inst. of Fund. Res. Studies in Math., no. 5, Oxford Univ. Press, London, 1970. MR 44 \#219. York, 1974.

[M2] - Prym varieties. I, Contributions to Analysis, Academic Press, New

[M3] Theta characteristics of an algebraic curve, Ann. Sci. Ecole Norm. Sup. (4) 4 (1971), 181-192. MR 45 \#1918.

DEPARTMENT OF MATHEMATICS, STATE UNIVERSITY OF NEW YORK AT STONY BROOK, STONY BROOK, NEW YORK 11790

Current address: Department of Mathematics, University of California, Irvine, California 92664 